\title{
Atención médica ambulatoria en México: el costo para los usuarios
}

\author{
Armando Arredondo, M.C., M. en C ., Ph.D., ${ }^{(1)}$ \\ Patricia Nájera, Lic. en Geogr., M. en C., (2) René Leyva, M.C ., M. en C. ${ }^{(1)}$
}

\section{Arredondo A, Nájera P, Leyva R. Atención médica ambulatoria en México: el costo para los usuarios. Salud Publica Mex 1999;41:18-26.}

\begin{abstract}
Resumen
Objetivo. Analizar los resultados de la Encuesta $\mathrm{N}$ acional de Salud II (EN SA-II), en lo relativo a los costos del proceso de búsqueda y obtención de la atención médica ambulatoria en diferentes instituciones del sector público y privado. Material y métodos. La informacion se obtuvo a partir de los indicadores de costos de la atención médica que notificó la población de estudio de la EN SA-II. Los costos para el bolsillo del consumidor fueron la variable dependiente, y las independientes, la condición de aseguramiento y el ingreso económico. La significancia de los niveles de variación se identificó aplicando la prueba de Duncan. Resultados. Los costos en todo el país, en dólares estadunidenses, fueron: transporte, \$2.20; consulta general, \$ 7.90; medicamentos, $\$ 9.60$, y estudios de diagnóstico, $\$ 13.6$. El costo promedio total de la atención ambulatoria fue de $\$ 22.70$. Los hallazgos empíricos permiten sugerir una nueva pro puesta de análisis de los costos en salud, tanto directos como indirectos, en que incurren los consumidores de servicios de salud; dichos costos representan una carga importante en relación con el ingreso familiar, situación que se agudiza en el caso de la población no asegurada. Conclusiones La incorporación de la perspectiva económica en el análisis de los problemas de los sistemas de salud, no debe limitarse a los costos de producción de servicios en que incurren los proveedores, sobre todo si lo que se busca es resolver los problemas de equidad y accesibilidad que actualmente caracterizan a la oferta de servicios médicos en México.
\end{abstract}

Palabras clave: costos de la atención en salud; accesibilidad a los servicios de salud; México

\author{
Arredondo A, Nájera P, Leyva $R$. \\ A mbulatory medical care in Mexico: \\ the cost for users. \\ Salud Publica Mex 1999;41:18-26.
}

\begin{abstract}
A bstract
Objective. To analyze the results of the $\mathrm{N}$ ational $\mathrm{Health}$ Survey (EN SA-II) as to the costs generated by the search and obtainment of ambulatory medical attention in various intitutions of the private and public health sector. Material and methods. Information was raised from the health care cost indicators reported by the study population of the EN SAII. The dependent variable was the direct expense for the consumer and the independent variables, the condition of being insured and the income. Variation significance levels were identified using the test by D uncan. Results. The costs at national level in US dollar were: transport \$2.20, medical visit $\$ 7.90$, drugs $\$ 9.60$, diagnostic studies $\$ 13.6$; average total cost for ambulatory attention was $\$ 22.70$. Empirical finding suggest a new direct and indirect cost-for-consumer analysis for the health care users. These costs represent an important burden on the family income, which worsens when users are not insured. Conclusions. Incorporation of the economic perspective to the analysis of public health issues should not be limited to the analysis of the health provider's expenses, particulary if the problems of equity and accessibility must be solved, which are at present characteristic of health care services in Mexico.
\end{abstract}

Key words: health care costs; health services accessibility; Mexico

(1) Centro de Investigación en Sistemas de Salud, Instituto N acional de Salud Pública (IN SP), México.

(2) Centro de Investigación en Salud Poblacional, IN SP, México.

Fecha de recibido: 15 de enero de 1997 - Fecha de aprobado: 18 de noviembre de 1998

Solicitud de sobretiros: Dr.Armando Arredondo. Centro de Investigación en Sistemas de Salud, Instituto N acional de Salud Pública. Av. Universidad 655, colonia Santa María A huacatitlán, 62508 Cuernavaca, Morelos, México. Correo electrónico: aarredon@ insp3.insp.mx 
D esde hace algunos años se ha incorporado la perspectiva económica como una herramienta potencial para entender y actuar sobre la producción y el financiamiento de los servicios de salud. ${ }^{1}$ En investigaciones recientes sobre el sector salud, en los planos internacional, nacional, regional y local, el análisis económico ha pasado a ocupar un lugar relevante; no sólo se han incluido variables de análisis económico, sino que se han desarrollado estudios donde dicho análisis es el objeto de trabajo y el hilo conductor para plantear problemas y soluciones a fin de mejorar el desempeño de los sistemas de salud. ${ }^{2}$

Para el caso de México, en los últimos años se ha realizado un importante esfuerzo para satisfacer las necesidades de salud de la población. Sin embargo, a pesar de los esfuerzos normativos instrumentados en la organización actual de los servicios de salud, ${ }^{3,4}$ existe una falta de consenso inter e intrainstitucional en materia de técnicas y procedimientos, de manera que es difícil establecer parámetros para medir productividad, costos y procedimientos de interacción por niveles de atención. Lo anterior se traduce en un sistema de salud cuyas normas son insuficientes para conducir y evaluar la eficiencia, en tanto no se cuente con la información necesaria. ${ }^{5,6}$

En relación con los costos de la atención médica en México, se presenta una situación muy peculiar. La organización de cada institución de salud plantea la necesidad de dos líneas de análisis: por un lado, los costos de producción de los servicios para el proveedor y, por el otro, los costos de búsqueda y obtención del servicio para el consumidor. Esta segunda línea de análisis es la que ha sido escasamente desarrollada en México y que se pretende abordar en el presente artículo; el análisis se limitará al costo de servicios ambulatorios, definidos como toda demanda de atención médica que no requiere de servicios de hospitalización. En este trabajo se presentan y discuten los resultados de la Encuesta Nacional de Salud II (ENSA-II) sobre costos de servicios ambulatorios, desglosados en costos de transporte, consulta y medicamentos. Estos hallazgos se contrastan con un marco conceptual que pretende identificar los diferentes eventos que se presentan durante el proceso de búsqueda y obtención de la atención médica.

\section{Consideraciones concept uales}

\section{Concepto de costos}

Trátese del consumidor o del proveedor, el concepto económico de costos surge de la noción de usos alternativos de los recursos. El costo económico de la uni- dad de un recurso es el beneficio que se obtendría de un mejor uso. Este concepto debe contrastarse con el concepto estrictamente contable de costo que está relacionado con las salidas de caja por unidad de recurso (gasto), o bien, con el costo de un bien o servicio que se establece en función del punto de equilibrio entre la oferta y la demanda (precio). Las estimaciones contable y económica de costos pueden coincidir, aunque de manera poco frecuente, pues no se incluye en la perspectiva contable el concepto de costo de oportunidad, y generalmente sólo se incluye lo referente a costo recurrente. Para el caso de los servicios de salud, y para fines de este análisis, lo importante es considerar que los costos se definen como el monto de recursos económicos que los usuarios desembolsaron durante cualquier evento del proceso de atención a la salud.7.8

Mills ${ }^{9}$ desarrolló una propuesta conceptual para el análisis de costos de los servicios de salud que considera los conceptos de costo-oportunidad, costos promedio, costo total y costo marginal. Además, plantea una tipología, que para fines de obtención y análisis, clasifica los costos en directos (aquellos en los que incurre directamente el proveedor de la atención médica para la generación de un servicio) e indirectos (aquellos en los que incurre el consumidor para obtener el servicio de atención médica, incluyendo tiempo de traslado, tiempo de espera, tiempo de tratamiento, etc.). ${ }^{10}$ Para el caso de este análisis, y en particular para el caso de México, esta propuesta teórica no es la idónea, ya que hay un costo directo tanto para el proveedor al momento de producir el servicio, como para el consumidor al momento de generar un desembolso económico por el consumo de servicios médicos. Es decir que en el contexto mexicano, los costos directos también se aplican al consumidor y no sólo al proveedor. En efecto, en el proceso de búsqueda y obtención de atención médica, procedente del sector público o del privado, los consumidores desembolsan un gasto que, para fines de este análisis, representa un costo directo al momento de utilizar un servicio, incluso en el caso de los asegurados, cuando deciden atenderse en instituciones de asistencia pública o privada.

De acuerdo con la clasificación descrita anteriormente para el análisis de costos de salud, para el caso de México se propone la misma clasificación pero con algunos ajustes:

Costos directos. Incluyen todo costo monetario en que incurre el proveedor en la generación del proceso de atención médica y todo desembolso del consumidor para la obtención de la misma.

Costos indirectos. Incluyen todo costo asociado al tiempo, dinero y esfuerzo, por parte del consumidor, du- 
rante la espera, el traslado y el periodo de tratamiento en el proceso de búsqueda y obtención de la atención médica.

Los costos y la búsqueda y obtención de la atención médica

Como propone Donabedian, ${ }^{11}$ el proceso de atención médica se concibe primordialmente como dos cadenas de actividades y procesos en los que participan, en forma paralela pero no inconexa, el proveedor de la atención médica, por una parte, y el consumidor, por la otra. Las actividades que constituyen el proceso de atención médica surgen como respuesta a una necesidad que generalmente se percibe como una alteración de la salud o del bienestar. En algunos casos el profesional de la salud puede ser el primero en detectarla, lo cual puede desencadenar el proceso que lleva a la atención médica.

El proceso de búsqueda de la atención lleva a establecer contacto directo con el proveedor. En algunos casos el camino que lleva hacia el médico es la información propia, o bien, la intervención de amigos y familiares para constituir lo que Freidson ${ }^{12}$ denomina "sistema no profesional de referencias". Esas actividades son el proceso de diagnóstico y toma de decisiones, y el proceso terapéutico en el cual interviene un "sistema profesional de referencias" para establecer un determinado patrón en el uso de los servicios ${ }^{13}$ y en el costo que el usuario desembolsa por dicho uso.

Los dos ejes principales del proceso de búsqueda de atención médica, el comportamiento del proveedor y el comportamiento del consumidor convergen en la obtención de atención médica, misma que debería culminar en la satisfacción o neutralización de la necesidad que desencadena originalmente el proceso. Así entonces, podría sugerirse que el proceso de búsqueda y obtención de atención médica puede concebirse como un proceso dinámico y complejo en el que intervienen variables psicosociales y culturales propias de la población, y variables político-administrativas propias de los servicios, que ponen en contacto a ambos actores, con el propósito de satisfacer una condición de salud determinada. ${ }^{14}$

A partir de estas consideraciones conceptuales, se pueden ubicar las etapas del proceso de la atención médica descritas con los costos directos e indirectos en que incurre el consumidor de servicios de atención médica ambulatoria. Para fines del análisis de los hallazgos de la ENSA-II sobre desembolsos económicos del consumidor, de acuerdo con esos conceptos y partiendo de los dos tipos de costos mencionados en la sección anterior, se pueden identificar como directos: los costos del proceso de diagnóstico, los costos de la atención misma (consulta) y los costos de los medicamentos; y como costos indirectos a aquellos propios de la búsqueda de atención (costos de traslado).

\section{Material y métodos}

La metodología, el material y el marco muestral de la ENSA-II han quedado descritos de manera detallada en un artículo que se refiere a las bases conceptuales y metodológicas de dicha encuesta, y que se publicó en 1998. ${ }^{15}$ Para los fines de este artículo, se presentan solamente la descripción, la delimitación y el manejo de las variables de estudio, así como los procedimientos metodológicos de los análisis univariado y bivariado que se llevaron a cabo y sus respectivas pruebas estadísticas.

Variables independientes. Condición de aseguramiento, definida a partir del derecho a los servicios de seguridad social indicado por la población (asegurado/no asegurado); el ingreso promedio anual del hogar (que sumó los ingresos de la población económicamente activa incluida en el hogar y los ponderó de acuerdo con el número de ocupantes del hogar), y el tipo de institución de búsqueda y obtención de la atención, a saber: unidades de seguridad social (Instituto Mexicano del Seguro Social e Instituto de Seguridad y Servicios Sociales de los Trabajadores del Estado), unidades de la Secretaría de Salud (Ministerio de Salud), servicios privados (instituciones lucrativas) y otras instituciones (incluyó privadas de beneficiencia y otras públicas). Tanto los costos para el consumidor como los percentiles de ingreso se expresaron en dólares estadunidenses, considerando como tasa de cambio promedio la de junio de 1994, esto es, 3.50 pesos mexicanos por un dólar estadunidense. Respecto a la variable ingreso promedio anual, el promedio de ingresos fue imputado, con base en el ingreso promedio del hogar y como una nueva variable, a cada integrante del hogar; posteriormente se categorizó según su distribución por cuartiles, definiendo estos últimos según los siguientes puntos de corte: de 0 a 2 258; > de 2258 a 4 457; de > 4457 a 8914 , y > 8914 .

Variables dependientes. Incluyen los desembolsos monetarios directos del bolsillo del consumidor que, para fines de este análisis, se traducen en: costo del transporte a la unidad de atención, costo por la atención, costo de medicamentos, costo de exámenes de laboratorio y gabinete, otros costos y costo promedio total. Se incluyeron los costos directos e indirectos en los que incurre la población durante el proceso de búsqueda y obtención de la atención, específicamente los costos notificados por 3319 usuarios de servicios ambulato- 
rios de salud durante los 15 días previos a la aplicación de la encuesta (mayo-junio de 1994).

A partir del análisis univariado se eliminaron valores extremos (valores considerados fuera del rango observable) de los costos notificados, y mediante el análisis de varianza se analizaron las diferecias entre los costos promedio por tipo de institución. En el análsis bivariado se buscaron las relaciones de los costos con los atributos socioeconómicos de los sujetos de estudio. Las variables independientes se midieron a partir de la condición de aseguramiento y el ingreso promedio anual del hogar. Se analizaron las diferencias de medias de los costos en tres estratos: por tipo de institución, por condición de aseguramiento y por ingreso familiar. Posteriormente se identificó la significancia de los niveles de variación entre grupos, aplicando la prueba de Duncan.

\section{Resultados}

El número estimado de usuarios de servicios fue de 4683 673; de éstos, 2951159 pagaron algún costo por los servicios utilizados. Cabe recordar aquí que toda cifra monetaria expresada en este artículo es en dólares estadunidenses. El costo promedio de transporte resultó en \$2.20, mientras que el costo promedio en consulta fue de $\$ 7.99$. Los medicamentos de usuarios que obtuvieron una receta alcanzaron un costo promedio de $\$ 9.60$, y entre aquellos a los que se les solicitó algún tipo de análisis y/o estudio de laboratorio, el desembolso promedio fue de $\$ 13.60$ (cuadro I). Otros gastos adicionales relacionados con la atención ocasionaron desembolsos variables (de \$ 6.80 a \$ 24.28).
El costo global promedio de la atención ambulatoria fue de \$22.70. En relación con los costos indicados por los usuarios según la institución en la que solicitaron el servicio, se observó un costo mayor entre los servicios privados ( $\$ 45.70$ en promedio), seguido de los que brinda la SSA (\$17.10), de otras instituciones públicas para población no asegurada (\$12.10) y, finalmente, de los usuarios no asegurados que se atendieron en instituciones de seguridad social (\$ 8.60 en promedio) (cuadro I).

El costo promedio en transporte de $\$ 2.20$ representa una cifra elevada si se considera que representa alrededor de $50 \%$ de un salario mínimo diario. Por tipo de institución a la que se acude, los costos de traslado mostraron disparidades importantes entre los servicios de seguridad social y los privados: en los primeros el costo fue de $\$ 1.60$ en promedio, en tanto que para los privados fue de $\$ 2.80$. Los diferenciales observados son mayores al contrastar los desembolsos por el viaje entre usuarios de comunidades rurales respecto a los que hacen los residentes de zonas urbanas: son equivalentes a más del doble en el primer caso en relación con el segundo (\$4.11 y \$1.68, respectivamente).

Como se preveía, los costos de la consulta para los usuarios variaron de manera importante según la institución: \$ 0.7 en los establecimientos de seguridad social (este costo mínimo se explica por la atención que se dio a 154583 no asegurados, los que notificaron un desembolso hacia la institución), \$4.10 en las unidades de la SSA, \$20.40 en los servicios del sector privado y $\$ 3.60$ en los otros servicios públicos para la población abierta.

Los medicamentos representan para los usuarios un costo similar al de la atención misma; en promedio,

\section{Cuadro 1 \\ Servicios ambulatorios de salud por tipo de institución. Costos para el usuario. ENSA-II, MÉXICO, 1994}

\begin{tabular}{|c|c|c|c|c|c|c|c|c|c|c|}
\hline \multirow[t]{3}{*}{ Elemento de costo } & \multicolumn{10}{|c|}{ M edias* de costo en dólares estadunidenses según tipo de consumidor ${ }^{\ddagger}$} \\
\hline & Naciona & IC & $\begin{array}{r}\text { Segurida } \\
\text { socials }\end{array}$ & d IC & SSA & IC & Privada & IC & Otra & IC \\
\hline & \multicolumn{2}{|c|}{ ne $=2951159$} & \multicolumn{2}{|c|}{ ne $=154583$} & \multicolumn{2}{|c|}{ ne $=886686$} & \multicolumn{2}{|c|}{ ne= 1541985} & \multicolumn{2}{|c|}{ ne $=367905$} \\
\hline Transporte & 2.2 & $(2.20-2.24)$ & 1.6 & $(1.69-1.71)$ & 2.4 & $(2.48-2.52)$ & 2.8 & $(2.87-2.90)$ & 2.5 & $(2.40-2.57)$ \\
\hline Consulta general & 7.9 & $(7.90-8.00)$ & 0.7 & $(0.74-0.77)$ & 4.1 & $(4.08-4.22)$ & 20.4 & $(20.39-20.53)$ & 3.6 & $(3.62-3.72)$ \\
\hline Medicamentos & 9.6 & $(9.58-9.69)$ & 1.4 & $(1.42-1.46)$ & 9.1 & $(9.0-9.27)$ & 17.0 & $(17.06-17.14)$ & 6.4 & $(6.44-6.53)$ \\
\hline Diagnóstico & 13.6 & $(13.56-13.76)$ & 1.5 & $(1.55-1.60)$ & 17.6 & $(17.52-17.82)$ & 32.3 & $(32.14-32.53)$ & 31.2 & $(30.0-32.4)$ \\
\hline Total de costos & 22.7 & $(22.73-22.85)$ & 8.6 & $(8.50-8.68)$ & 17.1 & $(17.03-17.28)$ & 45.7 & $(45.73-45.98)$ & 12.1 & $(12.1-12.2)$ \\
\hline \multicolumn{11}{|c|}{ * Prueba de Duncan $(<.05)$} \\
\hline \multicolumn{11}{|c|}{$\begin{array}{l}\text { ₹ Tasa de cambio: } 3.5 \text { pesos por un dólar estadunidense } \\
\S \text { Usuarios no asegurados que se atendieron en instituciones de seguridad social }\end{array}$} \\
\hline \multirow{2}{*}{\multicolumn{11}{|c|}{$\begin{array}{l}\text { ¿ Usuarios no asegurados que se atendieron en instituciones de seguridad social } \\
\text { IC: Intervalo de confianza al } 95 \%\end{array}$}} \\
\hline & & & & & & \multicolumn{5}{|c|}{$\begin{array}{l}\text { IC: Intervalo de confianza al } 95 \% \\
\text { ne: población estimada }\end{array}$} \\
\hline
\end{tabular}


los usuarios pagaron \$9.60. Este costo varió de acuerdo con los sectores involucrados: para los usuarios que acudieron a instituciones de seguridad social fue de $\$ 1.40$; los usuarios que acudieron a la SSA pagaron $\$ 9.10$, mientras que los del sector privado realizaron un desembolso de \$17.00. Cabe destacar que estos resultados de costos de medicamentos se obtuvieron a partir de la media nacional y las medias institucionales, a diferencia de otros análisis procedentes de la ENSA-II en las cuales el costo de los medicamentos se obtuvo a partir de medianas por tipo de institución y región sociodemográfica.

Los costos de los servicios auxiliares de diagnóstico, como análisis de laboratorio o gabinete, son mayores a los de la consulta misma y a los de los medicamentos: $\$ 13.50$ en promedio. Al respecto, la variación máxima entre instituciones se observa entre los servicios de seguridad social, con $\$ 1.50$ en promedio, y en los servicios privados con $\$ 32.30$. Los promedios de costo en este rubro, de acuerdo con el tipo de consumidor, fueron de $\$ 17.60$ para los no asegurados y de $\$ 31.20$ para otros servicios públicos.

$\mathrm{Al}$ analizar la relación de los costos con el tipo de institución, la condición de asegurado y el nivel de ingreso anual del hogar, se puede observar que para costos de transporte, en el caso de la población no asegurada que se atendió tanto en la seguridad social como en la SSA, existe una relación inversa, de tal manera que a menor ingreso, mayor costo del traslado (cuadro II). Una relación similar se observa para el caso de los que se atendieron en el sector privado $\mathrm{u}$ otro tipo de institución. Llama la atención que, en el caso de los asegurados que se atendieron en instituciones de seguridad social, también exista dicha relación inversa entre ingreso anual y costo de traslado.

En el análisis de la relación entre costo de la consulta por tipo de institución, condición de asegurado e ingreso anual del hogar, independientemente de la condición de asegurados o no asegurados y del tipo de institución donde se dio la consulta, destaca el hecho de que es en el percentil de mayor ingreso donde se observaron los mayores costos, de manera que existe una relación directa entre estas dos variables: a mayor ingreso, mayor costo de la consulta. Unicamente en el caso de los asegurados que se atendieron en institución privada lucrativa no se observó esa relación (cuadro III).

En cuanto a la relación de los costos de estudios de diagnóstico con la condición de asegurado, tipo de institución e ingreso anual por hogar, en el caso de la población no asegurada que se atendió en instituciones de seguridad social se observa una relación inversa, de tal manera que a menor ingreso mayor costo de estudios de diagnóstico. Un fenómeno similar, aunque no tan claro, se observa en el caso de los no asegurados que se atendieron en la SSA. La misma relación aparece de manera irregular en el caso de aquellos sujetos que se atendieron en el sector privado $\mathrm{u}$ otro tipo de institución.

Respecto al tipo de institución-costo-ingreso-condición de asegurado, para todos los tipos de costos por servicio se observó una fuerte asociación con altos cos-

\section{Cuadro II \\ COSTOS DE TRANSPORTE POR TIPO DE INSTITUCIÓN, CONDICIÓN DE ASEGURAMIENTO

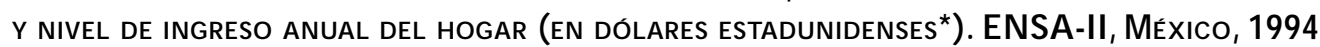

Percentil de ingreso

No asegurados

$0-2258 \$$

$>2258-4457 \$$

$>4457-8914 \$$

$>8914 \$$ Seguridad social
ne= 1572938 ne $=382362$
Privados

ne $=1026839$
Otros

ne $=229969$

Asegurados

\begin{tabular}{lrrrrr}
$0-2258 \$$ & $(26.1 \%)$ & 1.48 & 3.45 & 3.68 & 0.45 \\
\hline$>2258-4457 \$$ & $(6.4 \%)$ & 2.05 & 1.20 & 1.94 & 4.65 \\
\hline$>4457-8914 \$$ & $(6.6 \%)$ & 1.97 & 5.51 & 3.62 & 0.77 \\
\hline$>8914 \$$ & $(47.6 \%)$ & 1.05 & 1.20 & 1.57 & 0.54
\end{tabular}

*Tasa de cambio: 3.5 pesos por un dólar estadunidense

ne: población estimada

SSA : Secretaría de Salud 


\section{Cuadro III}

Costos de CONSULTA POR TIPO DE INSTITUCIÓN, CONDICIÓN DE ASEGURAMIENTO y Nivel de ingreso anUal del hogar (en dólares estadunidenses*). ENSA-II, MéXico, 1994

Percentil de ingresos

Seguridad social ne= 1950083

SSA
ne $=883890$

Privados

ne $=1518402$

Otros

$\mathrm{N} 0$ asegurados

\begin{tabular}{llllll}
$0-2258 \$$ & $(23.6 \%)$ & 2.74 & 4.20 & 17.20 & 1.68 \\
\hline$>2258-4457 \$$ & $(19.6 \%)$ & 1.54 & 4.45 & 15.91 & 7.31 \\
\hline$>4457-8914 \$$ & $(14.8 \%)$ & 0.71 & 4.77 & 18.37 & 5.77 \\
\hline$>8914 \$$ & $(11.2 \%)$ & 6.48 & 6.58 & 27.65 & 3.37
\end{tabular}

Asegurados

\begin{tabular}{lrrrrr}
$0-2258 \$$ & $(3.7 \%)$ & N A & 1.02 & 17.05 & 0.0 \\
\hline$>2258-4457 \$$ & $(8.0 \%)$ & N A & 2.14 & 18.25 & 1.37 \\
\hline$>4457-8914 \$$ & $(7.5 \%)$ & N A & 2.77 & 19.74 & 3.37 \\
\hline$>8914 \$$ & $(11.6 \%)$ & N A & 2.17 & 26.05 & 4.28
\end{tabular}

* Tasa de cambio: 3.5 pesos por un dólar estadunidense

ne: población estimada

N A: no aplica

SSA : Secretaría de Salud

tos en el sector privado. En el caso de los costos de medicamentos, para la misma relación de variables no se observaron asociaciones de relevancia, excepto la que se dio entre mayor costo y tipo de institución.

En la figura 1 se presentan los costos promedio nacionales sin diferenciar por tipo de institución ni por fases del proceso de la atención ambulatoria. En esa figura se esquematizaron las fases del proceso de búsqueda y obtención de la atención médica descritas en las consideraciones conceptuales, ubicando el costo promedio nacional que tuvo cada fase del proceso para la población en estudio. La integración del marco de referencia con los costos que resultaron de la encuesta permite identificar el peso relativo de cada fase del proceso. Así, es posible identificar que los costos de los estudios de diagnóstico y de los medicamentos son los de mayor peso relativo sobre el costo total del proceso estudiado. Por otra parte, un peso relativo menor correspondió a la búsqueda (transporte) de la atención y a la obtención de la misma. Estos hallazgos se retoman en la siguiente sección al hablar sobre sus consecuencias en aras de la accesibilidad y la equidad de los servicios de salud desde el proveedor hacia el consumidor, en México.

\section{Discusión}

Los hallazgos de la ENSA-II permiten hacer un análisis de alta representatividad, si se considera que, de acuerdo con el marco muestral, es posible la extra-

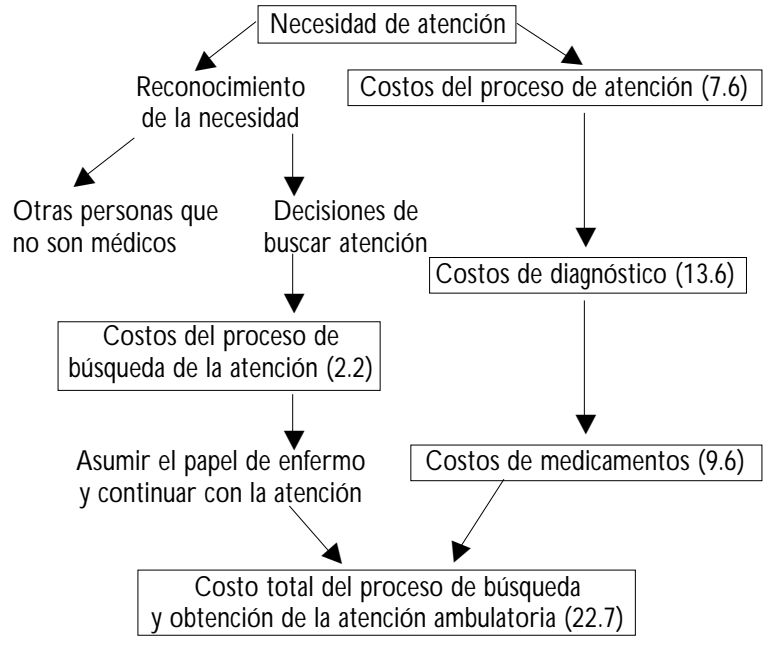

Tasa de cambio a junio de 1994:3.5 pesos por un dólar estadunidense

Figura 1. Costos para los usuarios en el proceso DE BÚSQUEDA Y OBTENCIÓN DE ATENCIÓN MÉdICA (PROMEDIO NACIONAL DE COSTOS POR EVENTO EN DÓLARES ESTADUNIDENSES). ENSA-II, MÉxico, 1994

polación a la población que consume los servicios de salud en México. En ese sentido se puede considerar que la siguiente discusión y las conclusiones son aplica- 
bles en todo el país. Por otra parte, tomando en cuenta que toda la información económica ha sido expresada en dólares estadunidenses, las cifras absolutas se pueden aplicar no sólo al periodo durante el cual se llevó a cabo la encuesta (junio de 1994), sino a cualquier otro lapso utilizando tasas de cambio vigentes, sobre todo si se busca tener una idea aproximada de los mismos costos para periodos de referencia posteriores.

El hecho de que los costos de transporte resultaran mayores para la población que se atendió en la SSA que para aquella que cuenta con seguridad social, indica que, desde la dimensión económica de accesibilidad, la infraestructura de los servicios de salud de la SSA es menos accesible económica y geográficamente que aquella de la seguridad social. Este problema de accesibilidad depende también del carácter urbano o rural de la comunidad de origen, de tal manera que el costo de traslado en la población rural superó en más del doble al de la población urbana. Estos resultados llaman la atención porque las comunidades rurales son las que tienen el menor ingreso anual y las que están más desprotegidas por los mismos programas de salud y por sus condiciones de vida en general. Por otra parte, estos resultados ponen en cuestionamiento los extensos programas normativos y operativos sobre extensión de cobertura que se han establecido en los últimos años y que, en principio, están dirigidos al derecho de protección de la salud de las comunidades más desprotegidas. Como se ha podido observar en este estudio, es indudable que en materia de accesibilidad y equidad se siguen presentando problemas que es necesario resolver desde la organización de los servicios a fin de contrarrestar el efecto económico, que en este caso se presenta al momento de buscar el servicio requerido, sobre todo si lo que se busca es contar con un sistema de salud cuya accesibilidad sea menos costosa para la población de menores ingresos.

En relación con el costo de la consulta para la población, cabe destacar que el hecho de notificar un costo de consulta de dos salarios mínimos para el caso de la población sin seguridad social, también representa problemas de acceso económico para poblaciones cuyo ingreso corresponde a una cifra no mayor a un salario mínimo.

Respecto al costo para el paciente de los medicamentos y estudios de diagnóstico, la situación se agudiza pues, en ambos casos, se trata de un costo mayor al de la atención misma; en particular los estudios de diagnóstico casi duplican el costo de la atención. Asimismo, es necesario destacar que para la población que se atendió en la SSA (en su mayoría no asegurada) el costo de los medicamentos fue tres veces más elevado al de la atención médica, y el de los estudios de diagnóstico, cinco veces superior. A pesar de tratarse de atención ambulatoria, esa situación sigue poniendo de manifiesto los problemas de equidad y accesibilidad que se pueden derivar del análisis económico, particularmente en el caso de los costos de búsqueda y obtención de atención en la población no asegurada. En otras palabras, de acuerdo con los datos obtenidos y con referencia exclusivamente al sector público, fue justamente la población de menores ingresos la que debió pagar más por los medicamentos y estudios de apoyo, situación contraria a los principios de equidad y accesibilidad económica del sistema de salud mexicano.

Al comparar los resultados por tipo de institución que brindó el servicio, se pueden apreciar diferencias sustanciales en los costos en que incurre el paciente para el consumo de un mismo servicio dependiendo de la institución donde se demandó la atención médica. Estas diferencias probablemente se deben a los distintos niveles de eficiencia y a los diferentes costos de producción con que cada institución opera. $\mathrm{Al}$ respecto, existe similitud con otros estudios nacionales e internacionales, cuyos hallazgos empíricos demuestran que al interior de cada institución se indican diferentes parámetros de eficiencia, de calidad de la atención, de combinación de insumos y de costos de los insumos. ${ }^{16-19}$ Los hallazgos de este análisis de costos confirman lo anterior, además de que ponen de manifiesto el efecto económico que tales diferencias institucionales tienen sobre el proceso de búsqueda y obtención de la atención médica. Otro aspecto que cabe destacar es que los resultados del costo de consulta externa en la SSA (\$14.40) están dentro del rango de costos $(\$ 13.00$ a $\$ 44.90$ ) determinados por otros estudios para la misma institución..$^{4,17}$ En el caso del sector privado el costo por consulta externa rebasa el límite superior de dicho rango, lo cual es un hecho en la práctica del sector privado.

Respecto a la integración del análisis de costos en el proceso de búsqueda y obtención de atención médica-costos directos-costos indirectos para el consumidor de servicios, el esquema propuesto en la figura 1 permitió proponer una clasificación de costos de servicios de salud que difiere de la clásica propuesta internacional para identificar costos directos e indirectos. En efecto, en esta propuesta los costos indirectos y directos son aplicables tanto a los fondos financieros del consumidor como a los del proveedor. Lo anterior es pertinente para el análisis económico de los problemas de equidad, accesibilidad y financiamiento de los servicios de atención médica bajo el sistema de salud mexicano por el hecho de que uno de los elementos que guían la propuesta de reforma del sector salud en 
México, en materia de dichos problemas, es el poder adquisitivo de la población y cómo se ve reflejado éste en el pago que el consumidor está dispuesto a realizar al momento de consumir servicios de atención médica. Por lo tanto, al identificar los montos económicos que desembolsa cada tipo de población se podrán resolver con mayor certidumbre los retos actuales sobre equidad, accesibilidad y financiamiento de la salud en México.

Al contrastar el esquema de la figura 1 con los datos generados por la encuesta se puede sugerir que, durante el proceso de búsqueda y obtención de la atención, la población no dispone de información suficiente que le permita acudir al centro de atención que le corresponde por derecho o prioridad. Lo anterior se apoya en el hecho de que usuarios con bajo ingreso económico y sin seguridad social utilizaron y pagaron servicios a instituciones del sistema de seguridad social o del sector privado. La hipótesis que se desprende entonces es la siguiente: si una persona percibe gravedad en su estado de salud y no es derechohabiente de los servicios de seguridad social, o bien, carece del poder adquisitivo para solicitar servicios de salud privados, pero vive cerca de una unidad de la seguridad social o de una clínica privada, en general acudirá a la clínica de la seguridad social donde, por su condición de no asegurada, no se le pueden hacer estudios de diagnóstico gratuitos ni proporcionarle medicamentos, o bien, pagará todos los servicios privados, a pesar de que en esa misma comunidad existen los servicios de atención médica para no asegurados. Este es uno de los casos típicos donde los problemas de equidad y accesibilidad generan un incremento en los costos de la atención.

Lo anterior se basa en los hallazgos de la ENSA-II que destacan, una vez más, el hecho de que mientras los planificadores del actual sistema de salud en México delimitan normativamente una determinada área de afluencia poblacional por tipo de institución, esa norma se ve rebasada por la realidad que opera en el proceso de búsqueda y obtención de la atención por parte de la población. Así, se pone de manifiesto que la perspectiva sobre los costos para el consumidor de servicios de salud es una variable que debe incluirse con mayor frecuencia en el análisis económico, epidemiológico o de cualquier otra disciplina que permita acercarse más al conocimiento y las posibles soluciones de los problemas sustantivos que enfrenan los sistemas de atención médica en México.

Los resultados de la ENSA-II podrían utilizarse en la planeación de los servicios de atención médica, para contrarrestar los efectos de los problemas de equidad y accesibilidad que caracterizan en la actualidad a los subsistemas de salud en México. Tradicionalmente se utiliza la escasa información económica que se tiene en materia de costos de producción de los servicios desde la perspectiva del proveedor; a partir de los datos de costos de la ENSA-II también se cuenta con información relevante sobre costos al consumidor en el proceso de búsqueda y obtención de la atención, misma que, per se, constituye un insumo fundamental para hacer de la salud un servicio mas accesible y equitativo dentro del amplio programa de reformas actuales del sector salud en México.

Respecto a la formación de recursos humanos, se recomienda que los resultados de esta encuesta en materia de costos, la propuesta de clasificación para este tipo de microanálisis económico y el esquema donde se integró el proceso de búsqueda y obtención de la atención con el análisis de costos para la población constituyan elementos empíricos y teóricos de discusión y promoción, sobre todo en los programas de posgrado en economía de la salud; ello contribuiría a una formación de excelencia académica, pertinente con la problemática de salud pública que caracteriza a nuestro país, particularmente en lo que a la evaluación económica de los servicios de salud se refiere.

\section{Agradecimientos}

Los autores agradecen particularmente al doctor Mauricio Hernández Avila los comentarios valiosos que aportó para el desarrollo de este artículo.

\section{Referencias}

1. Arredondo A, Hernández P, Cruz C. Incorporación de la perspectiva económica en el análisis del sector salud: segunda parte. Cuad Med Soc de Chile 1993;34(1):33-44.

2. Mills A. Decentralization and accountability in the health sector from an international perspective: $W$ hat are the choices? Public Administration and Development 1994;14:281-292.

3. Frenk J, Lozano R, G onzález-Block MA et al. Proyecto Economía y Salud. Informe final. 2a. edición. México, D.F.: FU N SALUD, 1995:62-64.

4. Secretaría de Salud/Instituto Mexicano del Seguro Social/Instituto de Seguridad y Servicios Sociales de los Trabajadores del Estado/Sistema $N$ acional de Evaluación. Programa N acional de Acción. Informe de Seguimiento y Evaluación: 1991-1992. México, D.F.: SSA (Publicación de Seguimiento, núm. 5), 1992:3-7.

5. Secretaría de Salud. Paquete básico de intervenciones en salud. Costos de intervenciones. Informe final. México, D.F.: Subsecretaría de Servicios de Salud, 1994:22-48.

6. Musgrove P. The economic crisis and its impact on health and health care in Latin America and the Caribbean. Int J Health Serv 1990;17(3): 411-441.

7. Dunlop D, Zubkoff L. Inflación y comportamiento del consumidor. En: análisis de costos, demanda y planificación de servicios de salud.W ashington, D.C.: O PS-O MS, 1990:221-261. 
8. Arredondo $\mathrm{A}$, Hernández $\mathrm{P}, \mathrm{Cruz} \mathrm{C}$. Incorporación de la perspectiva económica en el análisis del sector salud: primera parte. Cuad Med Soc Chile 1992;33(2):121-130.

9. Mills A, Gilson L. Health economics for developing countries. Londres: London School of Hygiene and Tropical Medicine-University of London (Publication num.17), 1988:59-62.

10. D rummond M, Stoddart G. Principles of economic evaluation of health programmes. W orld Health Stat Q 1985;38:347-354.

11. D onabedian A. Aspectos de la administración de la atención médica. México, D.F.: Fondo de Cultura Económica, 1987.

12. Freidson E. Types of lay referral systems. En: Social construction of illness-profession of medicine. C hicago: University of C hicago Press, 1988:

292-295.

13. Hulka $B, W$ heat J. Patterns of utilization: Patient perspective. Medical Care Rev 1985;23(5):438-460.

14. Rosenstock JM.W hy the people use health services: Part two. Milbank Q 1966;44(3):385-393.
15. Grupo Coordinador y de Diseño Conceptual de la ENSA-II. Bases conceptuales y metodológicas de la EN SA-II. México 1994. Salud Publica Mex 1998;40(1):76-85.

16. Arredondo A, Damian T, D e Icaza E. Una aproximación al estudio de costos de servicios de salud en México. Salud Publica Mex 1995;37(5):1-9. 17. Jamison D, Mosley H, Measham A, Bobadilla J. D isease control priorities in developing countries. Nueva York: World Bank (O xford Medical Publications), 1993.

18. Banco Mundial. Informe sobre el desarrollo mundial 1993. Invertir en salud: indicadores del desarrollo mundial.W ashington, D.C .:BM,1993: 126-128.

19. Barnum $\mathrm{H}$, Kutzin J. Public hospitals in developing countries: Resource use, cost and financing. Baltimore:The Johns Hopkins University Press, 1993. 